УДК 66.011

DOI: https://doi.org/10.17308/sait.2021.2/3504

Поступила в редакцию 05.04.2021

Подписана в печать 19.07.2021

\title{
СИНТЕЗ ОПТИМАЛЬНОЙ СИСТЕМЫ ТЕПЛООБМЕНА ПРОЦЕССОВ РАЗДЕЛЕНИЯ МНОГОКОМПОНЕНТНЫХ СМЕСЕЙ
}

\author{
(c) 2021 А. А. Рыжова ${ }^{\bowtie}$, И. И. Емельянов, Н. Н. Зиятдинов
}

Казанский национальный исследовательский технологический университет ул. К. Маркса, 68, 420015 Казань, Республика Татарстан, Российская Федерация

\begin{abstract}
Аннотация. Рассмотрены вопросы оптимальной организации системы теплообмена установки первичной перегонки нефти ЭЛОУ АВТ. Для этого с целью выявления экономической целесообразности функционирующей системы теплообмена проведен пинч-анализ. В результате пинч-анализа были построены композитные кривые горячих и холодных потоков и большая составная кривая, позволившие определить предельно возможное количество рекуперируемой энергии технологическими потоками, тип энергоносителей по энергетическому уровню и значение оптимальной движущей силы, при которой обеспечивается минимум критерия оптимальности - суммарных приведенных и капитальных затрат. Результаты пинч-анализа показали наличие около 30 \% полезной энергии для рекуперации технологическими потоками установки.

Анализ методов синтеза оптимальных систем теплообмена показал, что одним из наиболее эффективных методов решения крупномасштабных задач является декомпозиционный подход, который подразумевает разбиение задачи синтеза на несколько подзадач меньшей размерности: задачи линейного программирования для минимизации энергетических затрат, задачи дискретно-непрерывного линейного программирования для минимизации числа теплообменного оборудования, задачи нелинейного программирования для определения минимальных суммарных приведённых затрат.

В результате использования алгоритма декомпозиционного синтеза, были найдены оптимальные структуры системы теплообмена, режимы работы входящих в их состав теплообменников и даны экономические оценки найденным структурам. Результаты синтеза оптимальных систем теплообмена показали возможность для снижения суммарных приведенных затрат на 40 \% при реорганизации функционирующей системы теплообмена. Анализ результатов синтеза схем с возможностью регенерации тепловой энергии показал возможность дополнительной экономии до 6377 тыс. у.е./г, что стало возможным вследствие использования излишков энергии для подогрева технологических потоков близлежащих установок.

Ключевые слова: тепловая интеграция, ЭЛОУ АВТ, пинч-анализ, рекуперация тепла, синтез систем, математическая модель.
\end{abstract}

\section{ВВЕДЕНИЕ}

Разработка энергоэффективных технологических систем является одним из приоритетных направлений устойчивого развития химических, нефтехимических и нефтеперабатывающих предприятий [1], что обусловлено сложившимися экономическими усло-

Рыжова Алина Альбертовна e-mail: alinagainullina0@yandex.ru виями и экологическими проблемами. Высокая себестоимость производимой продукции актуализирует вопрос поиска резервов для повышения энергоэффективности предприятий за счет тепловой интеграции технологических потоков и вторичного использования топливно-энергетических ресурсов, а также разработки новых методов и алгоритмов автоматизированного технологического проектирования, позволяющих синтезировать оптимальные по экономическому критерию

Контент доступен под лицензией Creative Commons Attribution 4.0 License.

The content is available under Creative Commons Attribution 4.0 License. 


\section{А. А. Рыжова, И. И. Емельянов, Н. Н. Зиятдинов}

системы теплообмена материально-тепловых потоков.

К числу наиболее высокоэнергоемких промышленных предприятий относятся нефтеперерабатывающие заводы (НПЗ). Не смотря на высокую глубину переработки нефти и наличие множества тепловых рециклов, вопрос оптимальной организации системы теплообмена остается открытым [2]. Это связано как с многовариантностью самой задачи проектирования, так и отсутствием в проектной практике опыта использования методов и средств цифрового моделирования и математического программирования. Так, по данным [3] известно, что на НПЗ вся тепловая энергия, генерируемая технологическими установками, используется лишь на 30-35 \%, а оставшаяся часть уходит с охлаждающей водой или воздухом, с дымовыми газами, рассеивается в окружающую среду. Кроме того, особенностью таких производств является то, что поставляемая на переработку сырая нефть может значительно отличаться по составу. Изменение состава нефти может приводить к увеличению потребления энергоресурсов в связи с необходимостью удовлетворений требований на качество и выход производимой продукции.

К основным направлениям, способствующих решению задач энергоресурсосбережения и повышения эффективности нефтеперерабатывающих производств относятся: синтез экономически оптимальных систем рекуперативного теплообмена и использование высокоэффективных теплообменных аппаратов; вторичное использование вспомогательных топливно-энергетических ресурсов; организация гибкой системы теплообмена, оптимизация стационарных и динамических режимов работы технологических установок $[4,5]$. Отметим, что поскольку современные НПЗ представляют собой систему установок, работающих на разных энергетических уровнях, то имеется возможность использовать избыток тепловой энергии высокотемпературной установки для охлаждения или нагревания технологических потоков как внутри самой установки, так установки НПЗ, работающей при более низкой температуре [6].
Поэтому необходимо проанализировать режимы работы установок НПЗ, особенно при изменяющихся параметрах сырьевых потоков с точки зрения возможности минимизации потребления энергоносителей. Для этого необходимо провести анализ эффективности функционирующей системы теплообмена установок и, в случае выявления энергетических резервов решить задачу синтеза оптимальной системы теплообмена (СТО) $[7,8]$.

\section{Анализ результатов предыдущих работ}

В настоящее время разработаны множество подходов и методов синтеза систем теплообмена. Методы тепловой интеграции основаны на трех подходах: эвристическом [9], термодинамическом [10], математическом программировании [11-23].

Эвристические правила характеризуются быстротой и простотой решения задачи, но не позволяют получить оптимальные решения, поскольку часто противоречат друг другу. Термодинамический подход позволяет определить предельно возможное количество рекуперируемой энергии, количество теплоты, которое необходимо отобрать от горячих потоков и передать холодным потокам, тип энергоносителей по энергетическому уровню $[10,12]$. Он дает хорошие результаты, но, к сожалению, не в полной мере некоторые экономические аспекты задачи, что не гарантирует оптимальности получаемого решения. Эти недостатки могут быть устранены с помощью методов математического программирования на основе строгих математических моделей теплообменного оборудования [13-22]. В этом случае задача синтеза оптимальной СТО приобретает вид задачи смешанного целочисленного нелинейного программирования [13-16], которая может быть формализована на основе суперструктуры, содержащей множество возможных схем систем теплообмена. Такие методы позволяют достичь лучших экономических результатов, но характеризуются высокими вычислительными затратами. Одним из способов снижения трудоемкости задачи является ее структурная или целевая декомпозиция на основе 
транспортной модели $[24,25]$, или модели с промежуточными пунктами [26]. В связи с этим задачу синтеза оптимальных СТО предлагается решать в несколько этапов.

\section{1. МЕТОДЫ И МАТЕРИАЛЫ ИССЛЕДОВАНИЯ}

Пусть имеется $M^{\mathrm{h}}$ горячих потоков $S_{i}^{\mathrm{h}}$, $\left(i=1, \ldots, M^{\mathrm{h}}\right)$ и $M^{\mathrm{c}}$ холодных потоков $S_{j}^{\mathrm{c}}$, $\left(j=1, \ldots, M^{\mathrm{c}}\right)$, имеющих расходы $F_{i}^{\mathrm{h}}, F_{j}^{\mathrm{c}}$ и начальные температуры $T_{i}^{\mathrm{h}, \text {,in }}, T_{j}^{\mathrm{c}, \text { in }}$, соответственно. Известно, какое количество теплоты $\Delta Q_{i}^{\mathrm{h}}$ необходимо отвести, чтоб охладить каждый горячий поток до заданной температуры $T_{i}^{\mathrm{h}, \text { out }}$. Также будет известно, какое количество теплоты $\Delta Q_{j}^{\mathrm{c}}$ необходимо подвести каждому холодному потоку, для того, чтоб нагреть его до заданной температуры $T_{j}^{\mathrm{c}, \text { out }}$.

Задачи синтеза оптимальной СТО формализуется следующим образом. Необходимо определить структуру СТО, включающей рекуперативные теплообменники, нагреватели и холодильники, найти площади поверхностей теплообмена рекуперативных теплообменников $A_{i j}$, холодильников $A_{i}$ и нагревателей $A_{j}$, значения нагрузок $Q^{\text {he }}, Q^{\text {reb }}, Q^{\text {col }}$, при которых суммарные приведенные капитальные и эксплуатационные затраты будут иметь минимальное значение.

Рассматриваемая задача оптимального синтеза системы теплообмена является задачей большой размерностью вследствие наличия в установке ЭЛОУ АВТ множества источников и стоков тепловой энергии. Поэтому метод интегрального синтеза [15] не подходит для решения данной задачи. Одним из наиболее эффективных методов решения крупномасштабных задач является декомпозиционный, подразумевающий разбиение исходной задачи синтеза на несколько подзадач меньшей размерности [27]. Такой прием позволяет снизить размерность задачи, что является преимуществом при решении задач синтеза промышленного масштаба [26]. Он включает в себя следующие этапы: 1) осуществляется минимизация энергетических затрат с использованием методов линейного программирования; 2) решается задача дис- кретно-непрерывного линейного программирования для определения сочетания пар обменивающихся теплом потоков с целью минимизации числа теплообменного оборудования; 3) определяются минимальные суммарные приведённые затраты путем решении задачи нелинейного программирования.

В декомпозиционном подходе минимизация количества теплообменников является ключевым шагом для определения оптимальной структуры и минимальной стоимости сети теплообмена. Основными инструментами для расчета минимального количества теплообменников являются транспортная модель смешанного целочисленного программирования или модель с промежуточными пунктами.

Исходную задачу теплоинтеграции обычно трудно решить для всей теплообменной системы из-за вычислительной сложности. Поэтому на первом этапе предварительно проводится деление на температурные интервалы композитных кривых в соответствии с рис. 1.

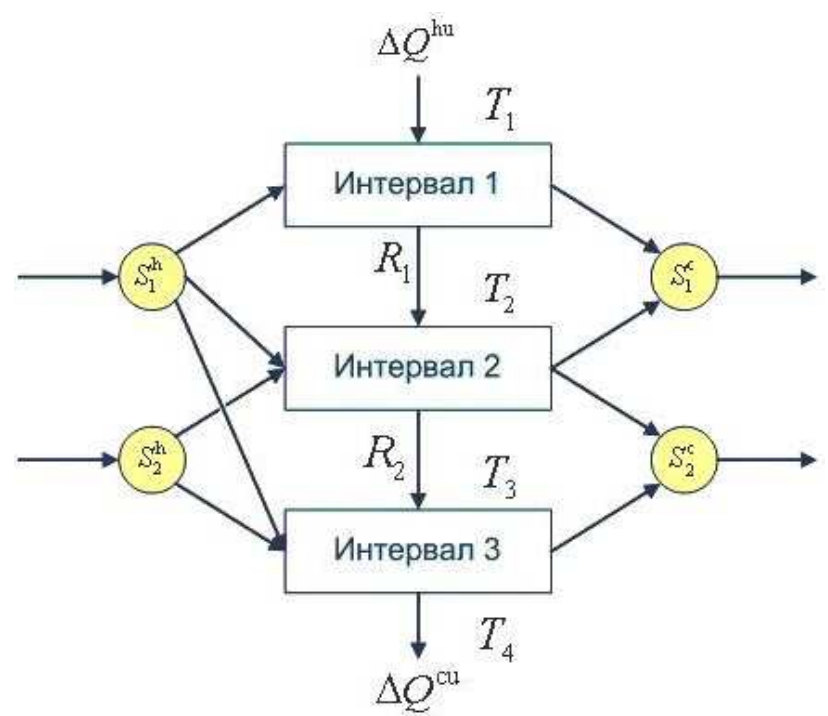

Рис. 1. Представление исходной задачи в виде температурных интервалов

[Fig. 1. Representation of the original problem in the form of temperature intervals]

Такое абстрагированное представление модели системы позволяет для решения этой задачи с целью минимизации потребления энергии использовать модель с промежуточными пунктами [25]: 


$$
\begin{gathered}
\min Z^{\prime}=\sum_{p \in \Omega} c_{p} \Delta Q_{p}^{\mathrm{hu}}+\sum_{t \in \Lambda} c_{t} \Delta Q_{t}^{\mathrm{cu}} ; \\
R_{i q}-R_{i,(q-1)}+\sum_{j \in C_{q}} \Delta Q_{i j q}+\sum_{t \in \Lambda_{q}} \Delta Q_{i t q}=Q_{i q}^{\mathrm{h}}, \\
\forall i \in \mathfrak{R}_{q}^{\prime}, \forall q \in K ; \\
R_{p q}-R_{p,(q-1)}+\sum_{j \in C_{q}} Q_{p j q}-Q_{p}^{\mathrm{hu}}=0, \\
\forall i \in \mathfrak{R}_{q}^{\prime}, \forall q \in K ; \\
\sum_{i \in \Re_{q}} \Delta Q_{i j q}+\sum_{p \in \Omega_{q}} \Delta Q_{p j q}=Q_{j q}^{c}, \forall j \in C_{q}, \\
\forall q \in K ; \\
\sum_{i \in \Re_{q}} \Delta Q_{i t q}-Q_{t}^{\mathrm{cu}}=0, \forall t \in \Lambda_{q}, \forall q \in K ; \\
R_{i q}, R_{p q}, Q_{i j q}, Q_{p j q}, Q_{i t q}, Q_{p}^{\mathrm{hu}}, Q_{t}^{\mathrm{cu}} \geq 0 ; \\
R_{i 0}=R_{i k}=0,
\end{gathered}
$$

где $K$ характеризует множество температурных интервалов; $c_{p}, c_{t}$ - удельные затраты на $p$-горячий и $t$-холодный теплоноситель, $\Delta Q_{i q}^{\mathrm{h}}$ и $\Delta Q_{j q}^{c}$ - количество теплоты, которое необходимо взять от $i$-горячего и передать $j$-холодному потоку на $q$-температурном интервале, соответственно; $\Delta Q_{p}^{\mathrm{hu}}$ и $\Delta Q_{t}^{\mathrm{cu}}-$ количество теплоты подводимое $p$-горячим и отбираемое $t$-холодным теплоносителем, соответственно; $\Delta Q_{i j q}, \Delta Q_{p j q}, \Delta Q_{i t q}$ - количество теплоты, передаваемое $i$-горячим потоком $j$-холодному, $p$-горячим теплоносителем $j$-холодному потоку, $i$-горячим потоком $t$-хладагенту на $q$-интервале, соответственно; $R_{i q}$ и $R_{p q}$ - остаточное количество теплоты $i$-горячего потока и $p$-горячего теплоносителя на выходе $q$-интервала. Пинч-зонами являются те интервалы, на выходе из которых остаточное количество теплоты равно нулю. Ниже представлен набор используемых индексов:

$\mathfrak{R}_{q}=\{i$-горячий поток отдаёт тепло на $q$-интервале $\}$

$\mathfrak{R}_{q}^{\prime}=\{i$-горячий поток находится на $q$ - или последующем интервале\};

$C_{q}=\{j$-холодный поток нуждается в тепле из $q$-интервала\};

$\Omega_{q}=\{p$-теплоагент отдаёт тепло в $q$-интервале\};

$\Omega_{q}^{\prime}=\{p$-теплоагент находится в $q$ - или последующем интервале\};

$\Lambda_{q}=\{t$-хладагент отбирает тепло из $q$-интервала\}.
На втором этапе с целью минимизации числа аппаратов для каждой подсети решается задача с промежуточными пунктами. Входными данными для ее решения являются полученные на первом этапе фиксированные значения тепловой энергии, отводимой хладоагентом и подводимой теплоносителем. Модель с промежуточными пунктами для каждой подсети принимает следующий вид:

$$
\begin{gathered}
\min \sum_{i \in \mathfrak{R}_{q}} \sum_{j \in C_{q}} y_{i j}^{\theta} \\
R_{i q}-R_{i,(q-1)}+\sum_{j \in C_{q}} \Delta Q_{i j q}=Q_{i q}^{\mathrm{h}}, \\
\forall i \in \mathfrak{R}_{q}^{\prime}, \forall q \in K_{\theta} \\
\sum_{i \in \mathfrak{R}_{q}} \Delta Q_{i j q}=Q_{j q}^{c}, \quad \forall j \in C_{q}, \forall q \in K_{\theta} ; \\
\sum_{q \in K_{\theta}} Q_{i j \theta}-Q_{i j}^{\mathrm{U}, \theta} y_{i j}^{\mathrm{U}, \theta} \leq 0, \\
\forall i \in \mathfrak{R}_{\theta}, \forall j \in C_{\theta} ; \\
R_{i q}, \quad Q_{i j q} \geq 0, y_{i j}^{\theta} \in\{0,1\},
\end{gathered}
$$

где индекс $K_{\theta}$ для каждого температурного интервала подсети $\theta ; y_{i j}^{\theta}-$ бинарная переменная, определяющая присутствие или отсутствие рекуперативного теплообмена между $i$-горячим и $j$-холодным потоками в подсети $\theta ; Q_{i j}^{\mathrm{U}, \theta}-$ верхняя граница количества теплоты, отбираемого от $i$-горячего потока и принимаемого $j$-холодным потоком в подсети $\theta$; индексы $\mathfrak{R}_{\theta}$ и $C_{\theta}$ применяются для всех горячих и холодных потоков в подсети $\theta$. Все остальные переменные и множества индексов имеют одно и то же значение, что и выше представленной модели. Верхняя граница $Q_{i j}^{\mathrm{U}, \theta}$ обычно определяется меньшим из общего содержания тепла $i$-горячего и $j$-холодного потоков в подсети $\theta$ :

$$
Q_{i j}^{\mathrm{U}, \theta}=\min \left\{\sum_{q \in K_{\theta}} Q_{i q}^{\mathrm{h}}, \sum_{q \in K_{\theta}} Q_{i q}^{\mathrm{c}}\right\} .
$$

На заключительном этапе с использованием модели нелинейного программирования решается задача минимизации капитальных затрат на полученной на предыдущем этапе структуре СТО, дополненной новыми связями до структуры вида, представленного на рис. 2. 


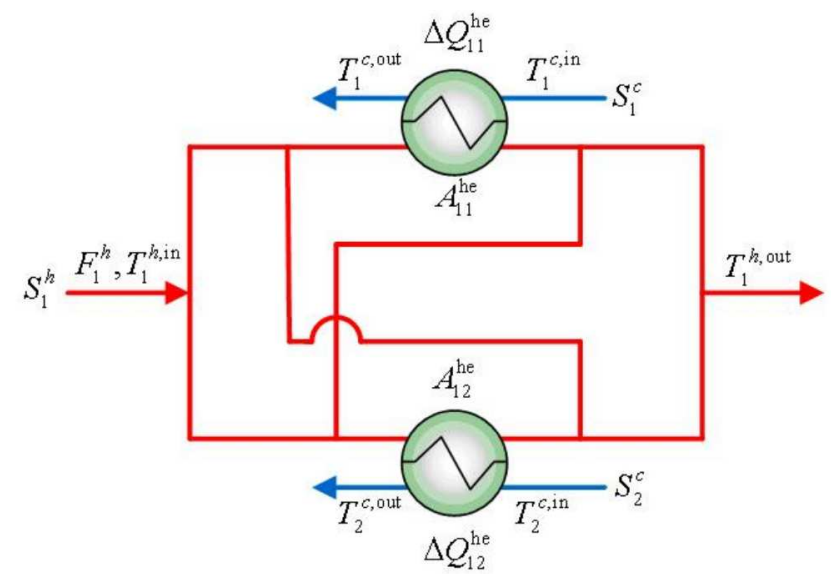

Рис. 2. Пример суперструктуры СТО для горячего и двух холодных потоков [15]

[Fig. 2. An example of HES superstructure for hot and two cold streams [15]]

\section{2. РЕЗУЛЬТАТЫ ИССЛЕДОВАНИЙ И ИХ ОБСУЖДЕНИЕ}

В качестве примера рассмотрена установка ЭЛОУ АВТ одного из крупнейших НПЗ, с целью выявления экономической целесообразности функционирующей системы те- плообмена. На рис. 3 представлена типовая технологическая схема данной установки.

Для решения задачи оптимальной теплоинтеграции была разработана компьютерная модель установки. При ее построении были использованы строгие математические модели технологического оборудования.

Построенная модель установки позволила рассчитать суммарные тепловые нагрузки рекуператоров, холодильников и нагревателей. Проведенный анализ показал, что суммарное количество рекуперируемой энергии системы составляет 133 МВт, суммарное количество энергии, подводимое к холодным потокам системы, оценивается на уровне 132 МВт, отводимое из системы - на уровне 91 МВт (табл.1).

Определим экономическую оценку существующей системы теплообмена установки ЭЛОУ АВТ по критерию суммарных приведенных капитальных и эксплуатационных затрат. Она складывается из капитальных затрат на рекуперативные теплообменники,

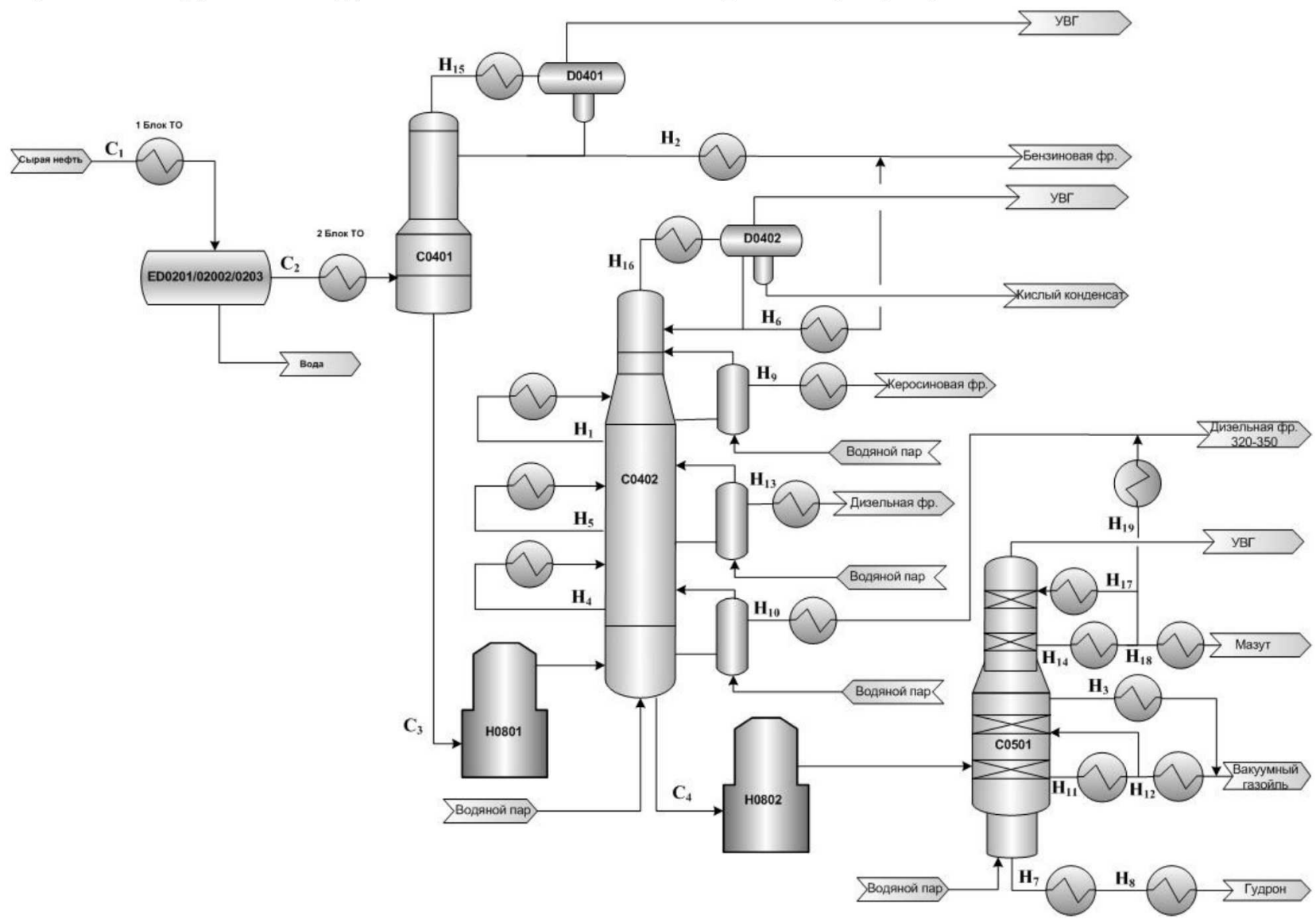

Рис. 3. Технологическая схема установки ЭЛОУ АВТ

[Fig. 3. Technological diagram of the CDU/VDU unit] 


\section{А. А. Рыжова, И. И. Емельянов, Н. Н. Зиятдинов}

нагреватели, холодильники и эксплуатационных затрат на подвод внешних холодных и горячих теплоносителей в холодильники и нагревателей, соответственно.

Таблииа 1. Результаты экономической оценки существующей системы теплообмена установки ЭЛОУ АВТ

[Table 1. Results of an economic assessment of the existing heat exchange system CDU/VDU units]

\begin{tabular}{|l|c|}
\hline \multicolumn{1}{|c|}{ Наименование показателя } & Значение \\
\hline Площадь рекуператора, ${ }^{2}$ & 13880 \\
\hline Площадь холодильника, м² & 32473 \\
\hline Площадь нагревателя, ${ }^{2}$ & 5728 \\
\hline Рекуперируемая энергия, МВт & 133 \\
\hline Отводимая энергия, МВт & 91 \\
\hline Подводимая энергия, МВт & 132 \\
\hline Количество рекуператоров, шт & 21 \\
\hline Количество холодильников, шт & 18 \\
\hline Количество нагревателей, шт & 2 \\
\hline Капитальны затраты, тыс. у.е./г & 5567 \\
\hline $\begin{array}{l}\text { Эксплуатационные затраты, } \\
\text { тыс. у.е. /г }\end{array}$ & 30365 \\
\hline $\begin{array}{l}\text { Суммарные приведенные } \\
\text { затраты, тыс. у.е. /г }\end{array}$ & 35931 \\
\hline
\end{tabular}

Эксплуатационные затраты рассчитываются на основе найденных с помощью компьютерной модели значений тепловых нагрузок теплообменного оборудования. Для расчёта капитальных затрат $K_{3}$ обычно используются аппроксимации следующего вида:

$$
\begin{gathered}
K_{3}=m_{1} A^{\gamma_{1}} \\
K_{3}=m_{2}+m_{3} A^{\gamma_{2}},
\end{gathered}
$$

где $m_{1}, m_{2}, m_{3}$ - ценовые коэффициенты; $A-$ площадь теплообмена; $\gamma_{1}, \gamma_{2}-$ показатель, величина которого варьируется в пределах $(0,6 \leq \gamma \leq 1)$.

В расчетах использовали аппроксимацию (15), так как данное уравнение хорошо аппроксимирует капитальные затраты для теплообменников небольших размеров, где также учитывается стоимость изготовления и монтажа аппаратов.

Учитывая линейный характер зависимости эксплуатационных затрат от количества отводимой/подводимой энергии с потоками энергоносителей расчеты проводились по формулам:

$$
\begin{aligned}
& E^{\mathrm{col}}=C_{t} \Delta Q^{\mathrm{col},}, \\
& E^{\mathrm{reb}}=C_{p} \Delta Q^{\mathrm{reb}}, \\
& E_{3}=E^{\mathrm{col}}+E^{\mathrm{reb}},
\end{aligned}
$$

где $E^{\mathrm{col}}, E^{\mathrm{reb}}-$ эксплуатационные затраты на холодильники и кипятильники; $E_{3}-$ общесуммарные эксплуатационные затраты; $C_{t}$, $C_{p}$ - стоимость 1 кДж отнимаемой/передаваемой энергии.

Расчет стоимости существующей системы теплообмена показал, что приведенные капитальные затраты составляют 5567 тыс. у.е./г, эксплуатационные затраты - 30365 тыс. у.е./г, суммарные приведённые капитальные и эксплуатационные затраты - 35931 тыс. у.е./г.

Для оценки термодинамического потенциала установки ЭЛОУ АВТ был проведен пинч-анализ потоков установки.

В качестве исходных данных использовалась информация о параметрах технологических потоков (табл. 2), полученных в результате расчета материльно-теплового баланса компьютерной модели установки ЭЛОУ АВТ.

На рис. 4а представлена температурно-энтальпийная диаграмма, из которой видна достаточно большая зона перекрытия горячей и холодной композитных кривых, характеризующая предельно возможное количество рекуперированной энергии. Это является хорошей предпосылкой для решения задачи оптимальной теплоинтеграции материально-тепловых потоков рассматриваемой установки. Исходя из характера композитных кривых можно сделать вывод о наличии равномерной движущей силы на большей части зоны перекрытия кривых, поэтому при проектировании системы теплообмена приобретает значение задача рационального выбора минимально допустимой разности температур. Очевидно, что её заниженное значение может значительно увеличить капитальные затраты на реализацию системы.

Зная значения ценовых коэффициентов с использованием методологии пинч-анализа можно оценить зависимость суммарных при- 
Синтез оптимальной системы теплообмена процессов разделения

Таблица 2. Холодные и горячие потоки установки ЭЛОУ АВТ [Table 2. Cold and hot streams of CDU/VDU unit]

\begin{tabular}{|c|c|c|c|c|c|c|c|}
\hline Потоки & $\begin{array}{c}\text { Входная } \\
\text { температу- } \\
\text { ра, } \mathrm{K}\end{array}$ & $\begin{array}{c}\text { Выходная } \\
\text { температу- } \\
\text { ра, } \mathrm{K}\end{array}$ & $\begin{array}{c}\text { Энтальпия, } \\
\text { МВт }\end{array}$ & Потоки & $\begin{array}{c}\text { Входная } \\
\text { температу- } \\
\text { ра, } \mathrm{K}\end{array}$ & $\begin{array}{c}\text { Выходная } \\
\text { температу- } \\
\text { ра, K }\end{array}$ & $\begin{array}{c}\text { Энтальпия, } \\
\text { МВт }\end{array}$ \\
\hline $\mathrm{H}_{1}$ & 456,5 & 368,1 & 20,69 & $\mathrm{H}_{14}$ & 479,1 & 333,2 & 23,87 \\
\hline $\mathrm{H}_{2}$ & 323,7 & 313,2 & 0,39 & $\mathrm{H}_{15}$ & 420,2 & 323,7 & 12,50 \\
\hline $\mathrm{H}_{3}$ & 533,0 & 363,2 & 7,25 & $\mathrm{H}_{16}$ & 382,6 & 324,3 & 43,12 \\
\hline $\mathrm{H}_{4}$ & 582,1 & 492,2 & 5,31 & $\mathrm{H}_{17}$ & 425,2 & 333,2 & 12,66 \\
\hline $\mathrm{H}_{5}$ & 539,1 & 447,2 & 18,42 & $\mathrm{H}_{18}$ & 425,2 & 313,2 & 1,00 \\
\hline $\mathrm{H}_{6}$ & 324,3 & 313,2 & 0,50 & $\mathrm{H}_{19}$ & 425,2 & 313,2 & 1,00 \\
\hline $\mathrm{H}_{7}$ & 642,0 & 549,8 & 22,34 & $\mathrm{FH}_{3}$ & 1273,0 & 673,1 & 77,58 \\
\hline $\mathrm{H}_{8}$ & 549,8 & 480,4 & 12,70 & $\mathrm{HP}_{2}$ & 522,1 & 523,1 & 1,32 \\
\hline $\mathrm{H}_{9}$ & 429,0 & 313,2 & 6,87 & $\mathrm{C}_{1}$ & 278,2 & 363,2 & 53,58 \\
\hline $\mathrm{H}_{10}$ & 541,1 & 313,2 & 2,38 & $\mathrm{C}_{2}$ & 372,3 & 501,8 & 80,04 \\
\hline $\mathrm{H}_{11}$ & 562,7 & 475,2 & 24,03 & $\mathrm{C}_{3}$ & 500,5 & 623,2 & 94,00 \\
\hline $\mathrm{H}_{12}$ & 440,8 & 345,3 & 7,58 & $\mathrm{C}_{4}$ & 609,2 & 668,2 & 37,23 \\
\hline $\mathrm{H}_{13}$ & 502,9 & 313,2 & 16,09 & $\mathrm{CU}$ & 293,1 & 303,1 & 37,71 \\
\hline
\end{tabular}

ведённых капитальных и эксплуатационных затрат от минимальной разности температур. Как видно из графика на рис. 46, прогнозируемый минимум суммарных приведённых затрат должен располагаться в интервале от $10{ }^{\circ} \mathrm{C}$ до $14^{\circ} \mathrm{C}$. Исходя из расчетов, минимально допустимую разность температур принимаем равной $10^{\circ} \mathrm{C}$.

Для определения типов и количеств подводимого и отводимого тепла от внешних горячих и холодных теплоносителей использовали большую композитную кривую (рис. 4в). Анализ кривой показал целесообразность использования следующих типов энергоносителей: вода оборотная $(\mathrm{CW})$, греющий пар высокого давления (HР) и печи (FH).

Проведенный пинч-анализ показал, что при выбранном значении минимальной движущей силы равном $10{ }^{\circ} \mathrm{C}$, предельно возможное количество рекуперируемой энергии системы составляет 186 МВт, при этом минимальное количество теплоты, подводимой горячими и отводимой холодными теплоносителями, составляет 79 и 38 МВт, соответственно.
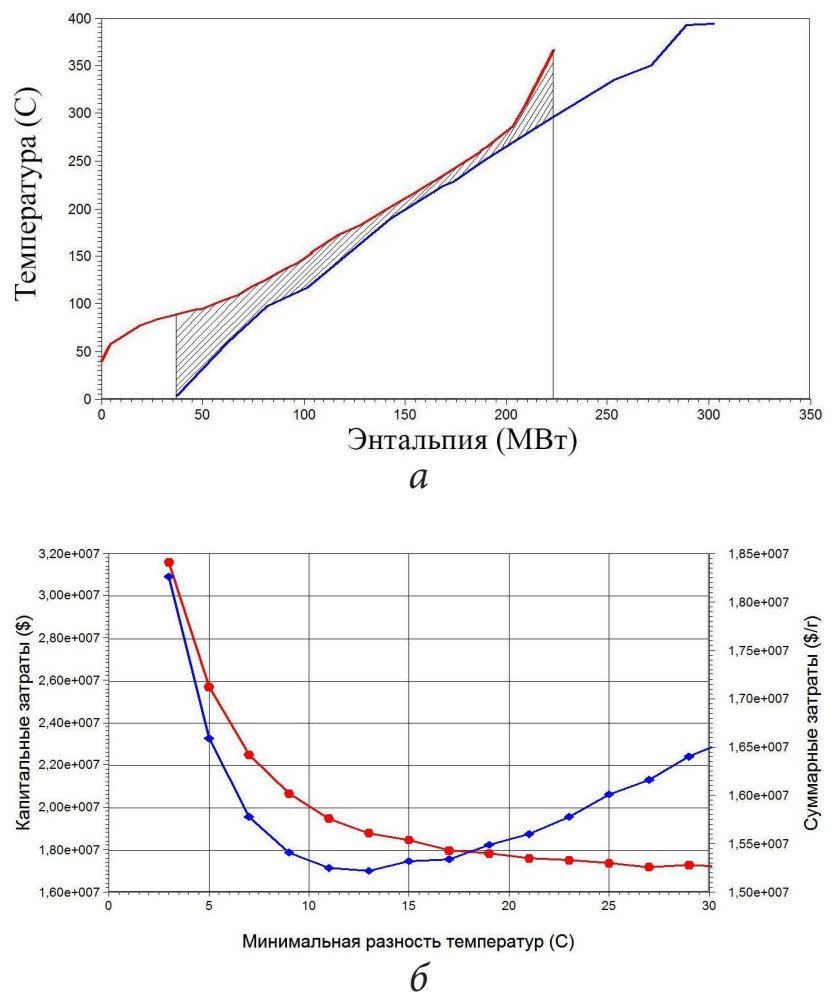


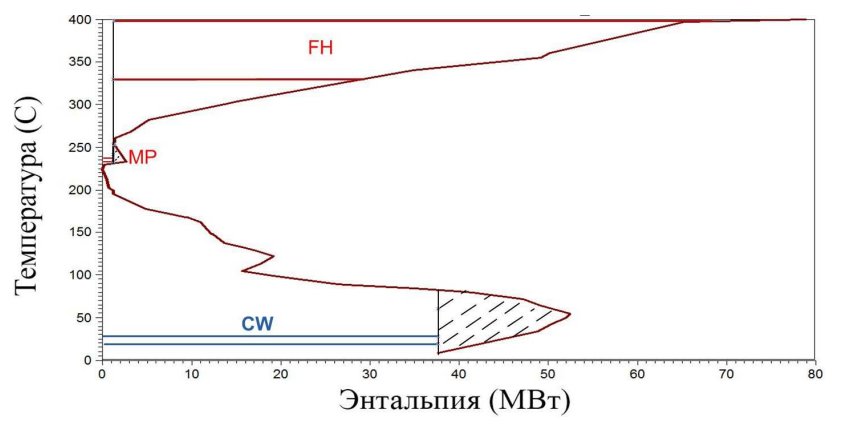

B

Рис.4. Результаты пинч-анализа:

а) композитные кривые; б) грабик зависимости капитальных и суммарных приведённых затрат от минимальной разности температур; в) большая композитная кривая

[Fig. 4. Pinch analysis results: a) composite curves; b) a graph of the dependence of capital and total reduced costs on the minimum temperature difference; c) large composite curve]

Если сравнить результаты полученные при помощи пинч-анализа с данными термодинамического анализа действующей установки ЭЛОУ АВТ (табл. 1), видно, что имеется около $30 \%$ неиспользованной тепловой энергии, которую можно применить для подогрева других потоков нефтеперерабатывающего комплекса. Следовательно, можно сделать вывод, что существующая система теплообмена малоэффективна и требуется синтез оптимальной СТО.

В результате использования алгоритма декомпозиционного синтеза, были найдены оптимальные структуры системы теплообмена и режимы работы входящих в их состав теплообменников (рис. 5).

В табл. 3 приведена экономическая оценка, полученных результатов синтеза оптимальной системы теплообмена.

Так же была рассмотрена возможность организации системы с учётом регенерации энергии, то есть реализация излишков энергоносителей в виде пара низкого и среднего давления. Результаты расчетов представлены в табл. 4.

По полученным результатам можно видно, что при использовании схемы СТО с регенерацией энергии значительно снижаются приведённые затраты, а именно на 6377 тыс. у.е./г.
Таблица 3. Результаты синтеза оптимальной СТО установки ЭЛОУ АВТ

[Table 3. The results of the synthesis of the optimal HES of the CDU/VDU unit]

\begin{tabular}{|l|c|}
\hline \multicolumn{1}{|c|}{ Наименование показателя } & Значение \\
\hline Площадь рекуператоров, м2 & 49381 \\
\hline Площадь холодильников, м2 & 771 \\
\hline Площадь нагревателей, м2 & 3632 \\
\hline Рекуперируемая энергия, МВт & 186 \\
\hline Отводимая энергия, МВт & 38 \\
\hline Подводимая энергия, МВт & 79 \\
\hline Количество рекуператоров, шт & 45 \\
\hline Количество холодильников, шт & 10 \\
\hline Количество нагревателей, шт & 4 \\
\hline Капитальные затраты, тыс. у.е./г & 4679 \\
\hline $\begin{array}{l}\text { Эксплуатационные затраты, } \\
\text { тыс. у.е. /г }\end{array}$ & 16915 \\
\hline $\begin{array}{l}\text { Суммарные приведенные } \\
\text { затраты, тыс. у.е./г }\end{array}$ & 21594 \\
\hline
\end{tabular}

Таблица 4. Результаты расчетов схемы СТО с учётом регенерации энергии

[Table 4. Results of calculations of the HES taking into account energy regeneration]

\begin{tabular}{|l|c|}
\hline \multicolumn{1}{|c|}{ Наименование показателя } & Значение \\
\hline Площадь рекуператоров, м² & 30029 \\
\hline Площадь холодильников, м² & 5695 \\
\hline Площадь нагревателей, ${ }^{2}$ & 8497 \\
\hline Рекуперируемая энергия, МВт & 105 \\
\hline Отводимая энергия, МВт & 119 \\
\hline Подводимая энергия, МВт & 160 \\
\hline Количество рекуператоров, шт & 41 \\
\hline Количество холодильников, шт & 34 \\
\hline Количество нагревателей, шт & 7 \\
\hline Капитальные затраты, тыс. у.е./г & 4251 \\
\hline $\begin{array}{l}\text { Эксплуатационные затраты, } \\
\text { тыс. у.е. /г }\end{array}$ & 10966 \\
\hline $\begin{array}{l}\text { Суммарные приведенные } \\
\text { затраты, тыс. у.е. /г }\end{array}$ & 15217 \\
\hline
\end{tabular}


Синтез оптимальной системы теплообмена процессов разделения

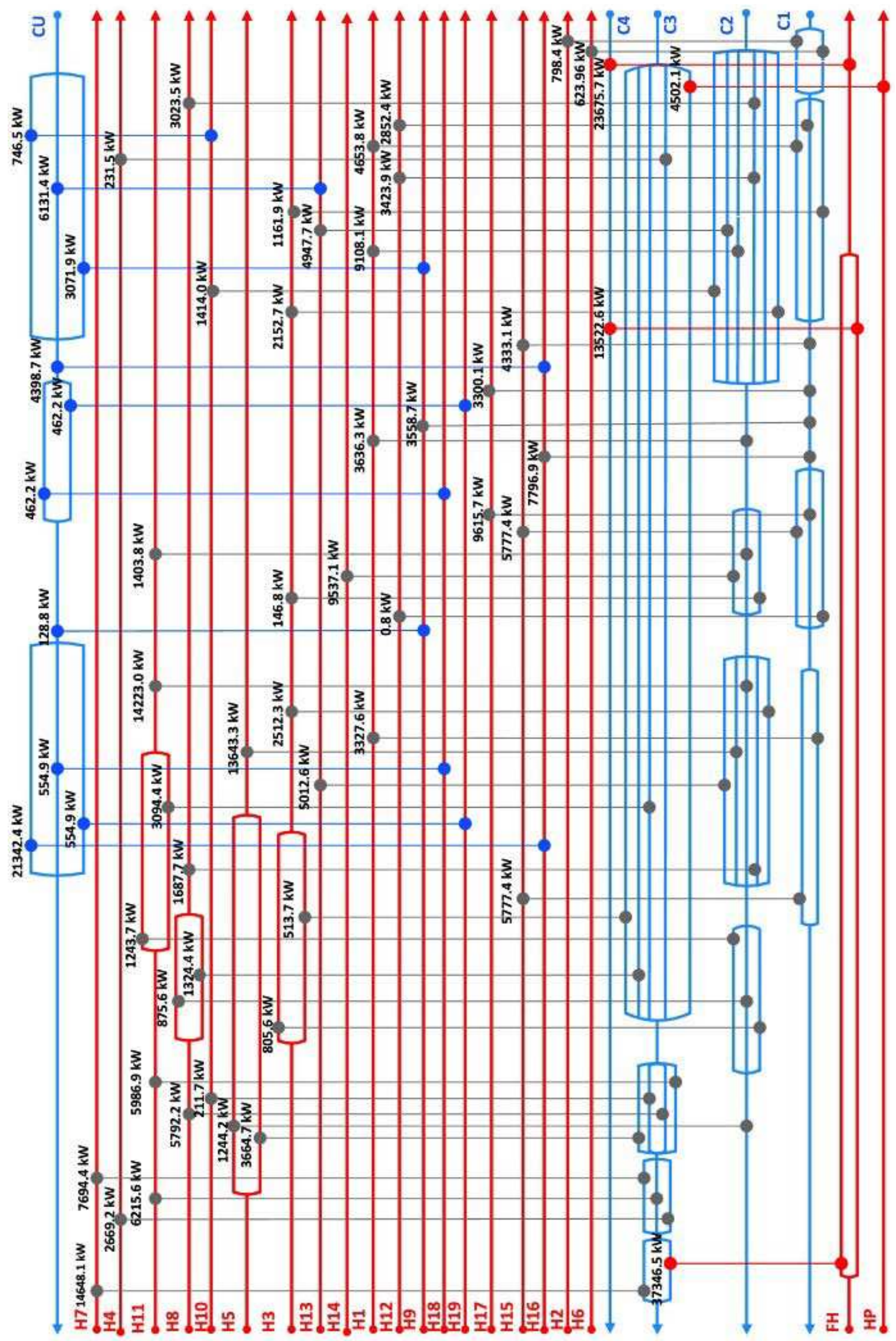

Рис. 5. Результаты синтеза оптимальной системь теплообмена

[Fig. 5. Results of synthesis of the optimal heat exchange system] 


\section{ЗАКЛЮЧЕНИЕ}

Проведенный экономический анализ установки ЭЛОУ АВТ показал низкую эффективность существующей системы теплообмена, которую можно объяснить следующими обстоятельствами:

1. Использование аппаратов воздушного охлаждения, которые сбрасывают полезную энергию в окружающую среду;

2. Нерациональным использованием низкопотенциальной энергии, которую можно направить для первоначального подогревания входного потока нефти;

3. Высокими движущими силами процесса теплообмена, которые хоть и снижают капитальные затраты, но приводят к увеличению суммарных приведенных затрат.

Результаты синтеза оптимальных систем теплообмена показали возможность снижения при реорганизации системы теплообмена суммарных приведенных затрат на $40 \%$. Анализ результатов синтеза схем с возможностью регенерации тепловой энергии показал возможность использования до 30 \% полезной энергии для подогрева других потоков нефтеперерабатывающего комплекса. Такое решение может дополнительно сэкономить до 6377 тыс. у.е./г.

\section{БЛАГОДАРНОСТИ}

Исследования выполнены в Казанском национальном исследовательском технологическом университете при поддержке Министерства науки и высшего образования РФ, грант № 075-00315-20-01 «Энергоресурсосберегающие процессы разделения жидких смесей для выделения промышленных растворителей».

\section{КОНФЛИКТ ИНТЕРЕСОВ}

Авторы декларируют отсутствие явных и потенциальных конфликтов интересов, связанных с публикацией настоящей статьи.

\section{СПИСОК ЛИТЕРАТУРЫ}

1. Boldyryev, S. Capital Cost Assessment for Total Site Power Cogeneration / S. Boldyryev, P. Varbanov, A. Nemet, J. Klemeš, P. Kapustenko // Computer Aided Chemical Engineering. 2013. - Vol. 32. - P. 361-366. DOI: 10.1016 / B978-0-444-63234-0.50061-0.

2. Чернышева, E. А. Проблемы и пути развития глубокой переработки нефти в России / Е. А. Чернышева // Бурение и нефть. - 2011. № 5.

3. Lisitsyn, N. V. Optimum control of a crude oil processing Plant / N. V. Lisitsyn, A. N. Krivospitskii, N. V. Kuzichkin // Theoretical Foundations of Chemical Engineering. 2002. - Vol. 36, No. 3. - P. 273-278. DOI: 10.1023/A:1015804122456.

4. Попов, А. П. Системный анализ и синтез предиктивной системы управления процессом термоокислительной деструкции полимера в реакторе периодического действия / А. П. Попов, С. Г. Тихомиров, И. А. Хаустов [и др.] // Вестник Воронежского государственного университета. Серия: Системный анализ и информационные технологии. - 2020. - № 1. C. 36-50. - DOI 10.17308/sait.2020.1/2582.

5. Тихомиров, С. Г. Программно-аппаратный комплекс для управления биотехнологическими системами с использованием интеллектуальных информационных технологий / С. Г. Тихомиров, И. А. Авцинов, Я. А. Туровский [и др.] // Вестник Воронежского государственного университета. Серия: Системный анализ и информационные технологии. - 2019. - № 3. - С. 158-165.

6. Song, $R$. Improved targeting procedure to determine the indirect interplant heat integration with parallel connection pattern among three plants / R. Song, Y. Wang, M. Panu, M. El-Halwagi, X. Feng // Ind. Eng. Chem.Res. - 2018. Vol. 57, No. 5. - P. 1569-1580. DOI: 10.1021 / acs. iecr.7b04327.

7. Tovazhnyanskii, L. L. Thermal process integration in the AVDU A12/2 crude distillation unit during winter operation / L. L. Tovazhnyanskii, P. A. Kapustenko, L. M. Ul'Ev, S. A. Boldyrev, O. P. Arsen'Eva, M. V. Tarnovskii // Theoretical Foundations of Chemical Engineering. - 
2009. - Vol. 43, No. 6. - P. 906. DOI: 10.1134 / S0040579509060086.

8. Bagajewicz, M. Retrofit of Crude Units Preheating Trains: Mathematical Programming versus Pinch Technology analysis (PA) and mathematical programming (MP) / M. Bagajewicz, G. Valitinson, D. Nguen Thanh // Ind. Eng.Chem. Res. - 2013. - Vol. 52, No. 42. - P. 14913-14926. DOI: 10.1021 / ie401675k.

9. Klemeš, J. J. New direction in the implementation of Pinch Methodology / J. J. Klemeš, P. S. Varbanov, T. G. Walmsley, X. Jia // Renewable and Sustainable Energy Reviews. - 2018. Vol. 98. - P. 439-468. DOI: 10.1016/j.rser. 2018.09.030.

10. Linnhoff, B. Synthesis of Heat Exchanger Networks. I. Systematic Generation of Energy Optimal Networks / B. Linnhoff, J. R. Flower // AIChE. - 1978. - Vol. 24. - P. 633-642. DOI: 10.1002/aic.690240411.

11. Biegler, L. T. Systematic methods of chemical process design / L. T. Biegler, I. E. Grossmann, A. W. Westerberg. - New Jersey : Prentice Hall PTR. - 1997.

12. Цирлин, А. М. Минимальная необратимость, оптимальное распределение поверхности и тепловой нагрузки теплообменных систем / А. М. Цирлин, А. А. Ахременков, И. Н. Григоревский // Теорет. основы хим. технологии. - 2008. - Т. 42, № 1. - C. 1. DOI: $10.1134 /$ S0040579508020139.

13. Biegler, L. T. Nonlinear Programming: Concepts, Algorithms, and Applications to Chemical Processes / L. T. Biegler // Philadelphia: Society for Industrial. Applied Mathematics, and Mathematical Optimization Society. - 2010. - P. 399. DOI: 10.1137 / 1.9780898719383.

14. Grossmann, I. E. Rigorous Disjunctive Optimization Model for Simultaneous Flowsheet Optimization and Heat Integration / I. E. Grossmann, H. Yeomans, Z. A. Kravanja // Comput. Chem. Eng. - 1998. - Vol. 22. - P. S157S164.

15. Yee, T. F. Simultaneous optimization models for heat integration - II. Synthesis of heat exchanger networks / T. F. Yee, I. E. Grossmann // Comput. Chem. Eng. - 1990. - Vol. 14, No. 10. P. 1165-1184. DOI: 10.1016 / 0098-1354 (90) 85010-8.
16. Емельянов, И. И. Синтез оптимальных одностадийных сетей теплообмена химико-технологических систем / И. И. Емельянов, Н. Н. Зиятдинов, Г. М. Островский // Вестник Технологического университета. - 2016. T. 19, № 17. - С. 132-137.

17. Лаптева, Т. В. Проектирование химико-технологических систем при учете объединенных мягких ограничений / Т. В. Лаптева, Н. Н. Зиятдинов, И. И. Емельянов // Теоретические основы химической технологии. 2020. - T. 54, № 1. - C. 17-29. DOI: 10.31857/ S0040357120010133.

18. Зиятдинов, Н. Н. Системный анализ установки ректификации этилового спирта как объекта теплоинтеграции / Н. Н. Зиятдинов, И. И. Емельянов, Г. З. Баймухаметова, Л. К. Туен // Вестник Технологического университета. - 2016. - Т. 19, № 15. - С. 131-136.

19. Ziyatdinov, N. N. Method of Automated Synthesis of Optimal Heat Exchange Network (HEN) Based on the Principle of Fixation of Variables / N. N. Ziyatdinov, I. I. Emel'yanov, T. V. Lapteva, A. A. Ryzhova, A. N. Ignat'ev // Theoretical Foundations of Chemical Engineering. -2020. - Vol. 54, No. 2. - P. 258-276. DOI: $10.1134 /$ S0040579520020189.

20. Ziyatdinov, N. N. Optimal heat exchanger network synthesis by sequential splitting of process streams / N. N. Ziyatdinov, I. I. Emel'yanov, Qi Chen, I. E. Grossmann // Computers \& Chemical Engineering. - 2020. - Vol. 142, No. 11. P. 107042-107090. DOI: 10.1016/j.compch emeng.2020.107042

21. Островский, Г. М. Синтез оптимальных систем простых ректификационных колонн с рекуперацией тепла / Г. М. Островский, Н. Н. Зиятдинов, И. И. Емельянов // Докл. АН. - 2015. - Т. 461, № 2. - 189 c. DOI: 10.7868/S0869565215080149.

22. Подвальньй, С. Л. Проблемы разработки интеллектуальных систем многальтернативного моделирования / С. Л. Подвальный, Т. М. Леденева, А. Д. Поваляев, Е. С. Подвальный // Вестник Воронежского государственного технического университета. - 2013. T. 9, № 3-1. - С. 19-23.

23. Подвальныцй, С. Л. Разработка специального программного обеспечения решения 
транспортных задач модифицированным генетическим алгоритмом с использованием многопоточности / С. Л. Подвальный, Д. А. Вдовин // Вестник Воронежского государственного технического университета. 2020. - T. 16, № 4. - C. 7-12. - DOI 10.25987/ VSTU.2020.16.4.001.

24. Cerda, J. Synthesizing heat exchanger networks having restricted stream/stream matches using transportation problem formulation / J. Cerda, A.W. Westerberg // Chem. Eng. Sci. - 1983b. - Vol. 10, No. 38. - P. 17231740. DOI:10.1016/0009-2509(83)85029-5

25. Cerda, J. Minimum Utility Usage in Heat Exchanger Network Synthesis / J. Cerda, A. W. Westerberg, D. Mason, B. Linnhoff //
Chem. Eng. Sci. - 1983. - Vol. 3, No. 38. - P. 373387. DOI: 10.1016 / 0009-2509 (83) 80156-0.

26. Papoulias, S. A. A Structural Optimization Approach in Process Synthesiss II. Heat Recovery Networks / S. A. Papoulias, I. E. Grossmann // Comput. Chem. Eng. - 1983. - Vol. 7, No. 6. P. 707-721. DOI: 10.1016/0098-1354(83)85023-6.

27. Барабанов, В. Ф. Декомпозиция задачи объемно-планировочного проектирования перерабатывающих предприятий / В. Ф. Барабанов, А. М. Нужный, С. Л. Подвальный // Вестник Воронежского государственного технического университета. - 2003. - № 8-3. C. 43-47.

Рыжова Алина Альбертовна - аспирант кафедры системотехники, Казанский национальный исследовательский технологический университет.

E-mail: alinagainullina0@yandex.ru

ORCID iD: https://orcid.org/0000-0001-5918-8296

Емельянов Илья Игоревич - канд. техн. наук, доц., доцент кафедры системотехники, Казанский национальный исследовательский технологический университет.

E-mail: ilyaemelyan@gmail.com

ORCID iD: https://orcid.org/0000-0003-0257-0739

Зиятдинов Надир Низамович - д-р техн. наук, проф., зав. кафедрой системотехники, Казанский национальный исследовательский технологический университет.

E-mail:nnziat@yandex.ru

ORCID iD: https://orcid.org/0000-0002-2314-8935 


\title{
SYNTHESISING AN OPTIMAL HEAT EXCHANGE SYSTEM FOR THE SEPARATION OF MULTICOMPONENT MIXTURES
}

\author{
(C) 2021 A. A. Ryzhova ${ }^{\bowtie}$, N. N. Ziatdinov, I. I. Emelyanov \\ Kazan National Research Technological University \\ 68, Karl Marx Street, 420015 Kazan, Republic of Tatarstan, Russian Federation
}

\begin{abstract}
Annotation. The article considers the issues of the optimal organisation of a heat exchange system for a CDU/VDU primary oil distillation unit. In order to determine the economic feasibility of the heat exchange system, a pinch analysis was performed. As a result of the pinch analysis we obtained composite curves of hot and cold flows and a large compound curve, which helped us to determine the maximum possible amount of energy recovered by the process flows, the type of energy carriers according to their energy level, and the optimal driving energy that ensures the minimum optimality criterion, i.e., the total of the operating and capital costs. The results of the pinch analysis showed that about $30 \%$ of the energy can be recovered by the process flows of the distillation unit.

Analysis of methods for the synthesis of optimal heat exchange systems demonstrated that the decomposition method is one of the most effective for solving large-scale problems. It implies dividing the synthesis problem into several subproblems of lower dimension: linear programming problems to minimize energy costs, discrete-continuous problems of linear programming to minimize the amount of heat exchange equipment, and nonlinear programming problems to determine the minimum total reduced costs.

Using the decomposition-based synthesis algorithm we determined the optimal structures of the heat exchange system and the modes of operation of the heat exchangers and obtained the economic estimates for these structures. The results of the study demonstrated that the total reduced cost can be reduced by $40 \%$, if the functioning heat exchange system is reorganised. Heat exchange systems designed with the function of thermal energy recovery can also save up to 6377 thousand $\mathrm{cu} / \mathrm{year}$, which is possible due to the use of surplus energy for heating process flows of nearby units.
\end{abstract}

Keywords: thermal integration, CDU/VDU unit, pinch analysis, heat recovery, system design, mathematical model.

\section{CONFLICT OF INTEREST}

The authors declare the absence of obvious and potential conflicts of interest related to the publication of this article.

\section{REFERENCES}

\section{Boldyryev S., Varbanov P., Nemet A., Klemeš} J. \& Kapustenko P. (2013) Capital Cost Assessment for Total Site Power Cogeneration. Computer Aided Chemical Engineering. 32, P. 361-366. DOI: 10.1016 / B978-0-444-63234-0.50061-0.

Ryzhova Alina A.

e-mail: alinagainullina0@yandex.ru
2. Chernysheva E. A. (2011) Problemy i puti razvitiya glubokoy pererabotki nefti $\mathrm{V}$ Rossii [Problems and ways of development of deep oil refining in Russia]. Drilling and oil. (5). (in Russian).

3. Lisitsyn N. V. (2002) Optimum control of a crude oil processing Plant. Theoretical Foundations of Chemical Engineering. 36 (3), P. 273-278. DOI: 10.1023/A:1015804122456. (in Russian).

4. Popov A. P., Tikhomirov S. G., Khaustov I. A. et al. (2020) System analysis and synthesis of a predictive control system for the process of thermooxidative destruction of a polymer in a batch reactor. Bulletin of the Voronezh State University. Series: System analysis and information technology. (1). P. 36-50. DOI 10.17308 / sait.2020.1 / 2582. (in Russian). 
5. Tikhomirov, S. G., Avtsinov, I. A, Turovsky Ya. A. et al. (2019) Hardware and software complex for managing biotechnological systems using intelligent information technologies. Bulletin of the Voronezh State University. Series: System Analysis and Information Technology. (3), P. 158-165. (in Russian).

6. Song R., Wang Y., Panu M., El-Halwagi M. \& Feng X. (2018) Improved targeting procedure to determine the indirect interplant heat integration with parallel connection pattern among three plants. Ind. Eng. Chem.Res. 57 (5), P. 15691580. DOI: 10.1021/acs.iecr. 7b04327.

7. Tovazhnyanskii L. L., Kapustenko P. A., Ul'Ev L. M., Boldyrev S. A., Arsen'Eva O. P. \& Tarnovskii M. V. (2009) Thermal process integration in the AVDU A12/2 crude distillation unit during winter operation. Theoretical Foundations of Chemical Engineering. 43 (6), P. 906-917. DOI: 10.1134 / S0040579509060086. (in Russian).

8. Bagajewicz M., Valitinson G. D. \& Nguen Thanh (2013) Retrofit of Crude Units Preheating Trains: Mathematical Programming versus Pinch Technology analysis (PA) and mathematical programming (MP). Ind. Eng.Chem.Res. 52 (42), P. 14913-14926. DOI: 10.1021 / ie401675k.

9. Klemeš J. J., Varbanov P. S., Walmsley T. G. \& Jia X. (2018) New direction in the implementation of Pinch Methodology. Renewable and Sustainable Energy Reviews. 98, P. 439-468. DOI: 10.1016/j.rser. 2018.09.030.

10. Linnhoff B. \& Flower J. R. (1978) Synthesis of Heat Exchanger Networks. I. Systematic Generation of Energy Optimal Networks. AIChE. 24, P. 633-642. DOI: 10.1002/aic. 690240411.

11. Biegler L. T. (1997) Systematic methods of chemical process design. In: Grossmann I. E., Westerberg A. W. New Jersey: Prentice Hall PTR.

12. Tsirlin A. M., Akhremenkov A. A. \& Grigorevskii I. N. (2008) Minimal irreversibility and optimal distributions of heat transfer surface area and heat load in heat transfer systems. Theoretical Foundations of Chemical Engineering. 42 (2), P. 203-210. DOI: 10.1134/S0040579508 020139. (in Russian).

13. Biegler L. T. (2010) Nonlinear Programming: Concepts, Algorithms, and Applications to Chemical Processes. Philadelphia: Society for Industrial. Applied Mathematics, and Mathema- tical Optimization Society. P. 399. DOI: 10.1137 / 1.9780898719383.

14. Grossmann I. E., Yeomans H. \& Kravanja Z. A. (1998) Rigorous Disjunctive Optimization Model for Simultaneous Flowsheet Optimization and Heat Integration. Comput. Chem. Eng. 22, S157-S164.

15. Yee T. F. \& Grossmann I. E. (1990) Simultaneous optimization models for heat integration - II. Synthesis of heat exchanger networks. Comput. Chem. Eng. 14 (10), P. 1165-1184. DOI: 10.1016 / 0098-1354 (90) 85010-8.

16. Emelyanov I. I., Ziyatdinov N. N. \& Ostrovsky G. M. (2016) Sintez optimal'nykh odnostadiynykh setey teploobmena khimiko-tekhnologicheskikh system [Synthesis of optimal one-stage heat exchange networks of chemical-technological systems]. Bulletin of the Technological University. 19(17), P. 132-137. (in Russian).

17. Lapteva T. V., Ziyatdinov N. N. \& Emel'yanov I. I. (2020) Chemical process design taking into account joint chance constraints. Theoretical Foundations of Chemical Engineering. 54(1), P. 145-156. DOI: 10.31857/ S0040357120010133. (in Russian).

18. Ziyatdinov N. N., Emelyanov I. I., Baimukhametova G. Z. \& Tuen L. K. (2016) System analysis of the ethyl alcohol rectification unit as an object of heat integration. Bulletin of the Technological University. 19 (15), P. 131-136. (in Russian).

19. Ziyatdinov N. N., Emel'yanov I. I., Lapteva T. V., Ryzhova A. A. \& Ignat'ev A. N. (2020) Method of Automated Synthesis of Optimal Heat Exchange Network (HEN) Based on the Principle of Fixation of Variables. Theoretical Foundations of Chemical Engineering. 54 (2), P. 258-276. DOI: 10.1134/ S0040579520020189. (in Russian).

20. Ziyatdinov N. N., Emel'yanov I. I., Chen Qi \& Grossmann I. E. (2020) Optimal heat exchanger network synthesis by sequential splitting of process streams. Computers \& Chemical Engineering. 142 (11), P. 107042-107090.. DOI: 10.1016 / j.compchemeng.2020. 107042

21. Ostrovskii G. M., Ziyatdinov N. N. \& Emel'yanov I. I. (2015) Synthesis of optimal systems of simple distillation columns with heat recovery. Doklady Chemistry. 461 (1). P. 89-92. DOI: 10.7868/S0869565215080149. (in Russian). 
22. Basement S. L., Podvalny S. L., Ledeneva T. M., Povalyaev A. D. \& Podvalny E. S. (2013) Problems of development of intelligent systems of multichannel modeling. Bulletin of the Voronezh State Technical University. 9 (3-1), P. 19-23. (in Russian).

23. Podvalny S. L. \& Vdovin D. A. (2020) Development of special software for solving transport problems with a modified genetic algorithm using multithreading. Bulletin of the Voronezh State Technical University. 16 (4), P. 7-12. DOI 10.25987 / VSTU.2020.16.4.001. (in Russian).

24. Cerda J. \& Westerberg A. W. (1983b) Synthesizing heat exchanger networks having restricted stream/stream matches using transportation problem formulation. Chem. Eng. Sci. 10 (38), P. 1723-1740. DOI:10.1016/00092509(83)85029-5.

Ryzhova Alina A. - postgraduate student. Department of Systems Engineering, Kazan National Research Technical University named after A. N. Tupolev - KAI.

E-mail: alinagainullina0@yandex.ru

ORCID iD: https://orcid.org/0000-0001-5918-8296

Emelyanov Ilya I. - PhD in Technical Sciences, Associate Professor, Department of Systems Engineering, Kazan National Research Technical University named after A. N. Tupolev - KAI.

E-mail: ilyaemelyan@gmail.com

ORCID iD: https://orcid.org/0000-0003-0257-0739

Ziyatdinov Nadir N. - DSc in Technical Sciences, Professor, Head of the Department of Systems Engineering, Kazan National Research Technical University named after A. N. Tupolev - KAI.

E-mail: nnziat@yandex.ru

ORCID iD: https://orcid.org/0000-0002-2314-8935
25. Cerda J., Westerberg A. W., Mason D. \& Linnhoff B. (1983) Minimum Utility Usage in Heat Exchanger Network Synthesis. Chem. Eng. Sci. 3 (38), P. 373-387. DOI: 10.1016 / 0009-2509 (83) 80156-0.

26. Papoulias S. A. \& Grossmann I. E. (1983) A Structural Optimization Approach in Process Synthesiss II. Heat Recovery Networks. Comput. Chem. Eng. 7 (6). P. 707-721. DOI: 10.1016/00981354(83)85023-6.

27. Barabanov V. F., Nuzhny A. M. \& Podvalny S. L. (2003) Decomposition of the problem of space-planning design of processing enterprises. Bulletin of the Voronezh State Technical University. (8-3), P. 43-47. 